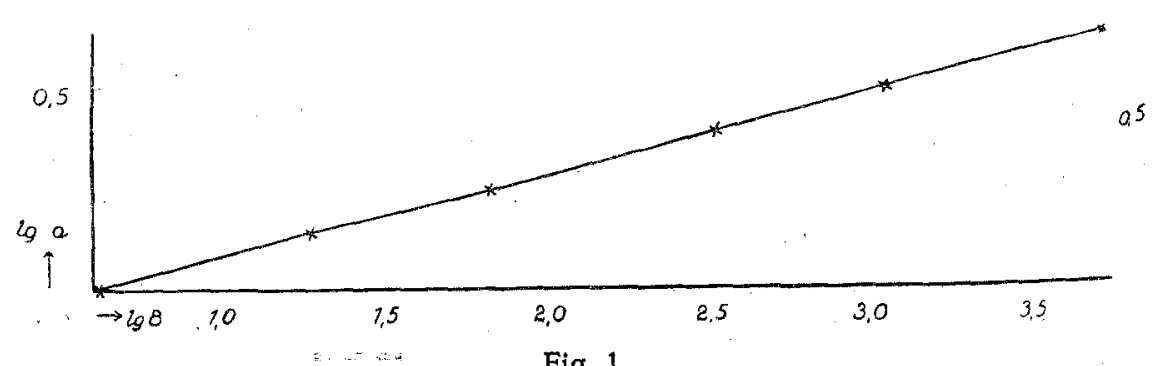

Fig. 1

nachlässigen konnte. Tabelle I zeigt die Koagulationswerte in Mikromolen im Liter, Fig. 1 die aus der Adsorptionsisotherme folgende Beziehung: Die Logarithmen der Koagulationswerte $\gamma$ der verschiedenwertigen Ionen als Abszissen den Logarithmen der $\alpha$-Werte 1, 1/2, $1 / 3,1 / 4,1 / 6$ - bzw. $6 ; 3 ; 2 ; 1,5 ; 1$ - als Ordinaten zugeordnet, sollten eine Adsorptionsisotherme ergeben, also auf einer graden Linie liegen. Fig. 1 zeigt, daß dies völlig zutrifft.
Unter den von mir kurz berührten Erscheinungen ist keine, bei der nicht $z u$ erwarten wäre, daß glücklich gewählte, weitere Fälle Wichtiges bestätigen oder wesentlich Neues zutage fördern würden.

Dahlem.

Kaiser-Wilhelm - Institut

für physikalische Chemie und Elektrochemie.

\title{
Die Kolloidchemie vom Standpunkt der Elektrochemie.
}

Von L. Michaelis (Berlin).

(Eingegangen aw 24. September 1922.)

Die Teilchen einer kolloiden Lösung haben im allgemeinen eine elektrische Potentialdifferenz gegenüber dem Lösungsmittel. Dieser Umstand allein genügt schon, um die Frage vorzulegen: „Welche Beziehung hat die Kolloidchemie zur Elektrochemie, in welcher ja auch von kleinen Teilchen, die gegenüber dem Lösungsmittel eine elektrische Ladung haben, nämlich von den lonen, die Rede ist?" Diese Analogie ist aber in letzter Zeit noch viel größer geworden, nachdem es sich gezeigt hat, daß wesentliche Eigenschaften der Ionen, insbesondere ihre Wanderungsgeschwindigkeit, theoretisch nach denselben Prinzipien behandelt werden können wie die Bewegung größerer Te:lchen in einem flüssigen homogenen Medium. Durch die theoretischen Untersuchungen von $\mathrm{E}$ in stein über den Zusammenhang der B rownschen Molekularbewegung mit der kinetischen Gastheorie wurde zum ersten Mal die Anwendung eines in der theoretischen Hydrodynamik altbekannten Gesetzes, nämlich des Stokes schen Gesetzes, auf so kleine Gebilde, wie es die dispersen Teilchen einer Kolloidlösung sind, ermöglicht. R. Lor e $z$ ist hierüber noch hinausgegangen und hat versucht, auch die Bewegungen einzelner Ionen nach dem St okesschen Gesetz abzuleiten, d. h. die Ionen als starre Kügelchen $\mathrm{zu}$ betrachten, welche in einer homogenen Flüssigkeit von einer gewissen Viskosität sich bewegen. Da nach dem Stokesschen Gesetz die Beweglichkeit eines kugelförmigen Teilchens von der Größe seines $\mathrm{Ra}-$ dius abhängt, versuchte Lorenz unter Anwendung des Stokes'schen Gesetzes die Beweglichkeit der lonen, welche aus verschiedenen Messungen bekannt ist, zu benutzen, um aus ihr die Größe der Ionen $z u$ berechnen. $\mathrm{Er}$ kam dabei überraschenderweise zu Werten derselben Größenordnung wie sie auf Grund ganz anderer Rechnungen gefunden wurden. Bei Ionen von besonders kleinem Radius ergaben sich hierbei Schwierigkeiten, welche jedoch durch die neuen Theorien von Born beseitigt scheinen. Ohne auf' die Einzelheiten dieser höchst interessanten Forschungen einzugehen, kann man behaupten, dab die elektrischen Erscheinungen kolloidzerteilter Teilchen genau unter demselben Gesichtspunkte zu behandeln sind, wie diejenigen der Ionen. Schon vor langer Zeit ist wiederholt die Idee ausgesprochen worden, daß in ihrem ganzen Verhalten kolloide Teilchen vergleichbar sind mit Ionen, nur daf sie viel gröBer sind und statt einer einzigen oder mehrerer einzelner Ladungen eine Menge von elektrischen Elementarladungen tragen. Die Teilchen einer kolloiden Lösung sind gewissermaßen Riesenionen, und 
ein solches Riesenion, in Gemeinschaft mit denjenigen entgegengesetzt geladenen Ionen der Lösung, welche mit ihm zusammen ein elektrisch neutrales Ganze ergeben, sind gewissermaßen nichts weiter als eine besondere Art von Elektrolyten. Die elektrische Kataphorese kolloider Teilchen erscheint somit in ihrem Wesen aufs engste verwandt mit der gewöhnlichen elektrolytischen Leitung der Lösungen, und die gewöhnliche elektrolyıische Leitung der Lösungen ist nur der Grenzfall, bei dem die geladenen Teilchen sämtlich molekulardispers, bzw. ionendispers sind, wenn ich mich dieses Ausdruckes von Wo. Ostwald bedienen soll Der Zusammenhang der Kolloidchemie und Elektrochemie ist daher sehr eng. Das Problem der Zukunft ist es offenbar, die Gesetze der elektrischen. Kataphorese in einer derart allgemein gültigen Form auszusprechen, daß die Gesetze der gewöhnlichen elektrischen Leitfähigkeit der Lösungen als Grenzfall für molekulare Dispersion von selbst daraus hervorgehen. Hiervon sind wir allerdings noch weit entfernt, und es soll meine heutige Aufgabe sein, unser heutiges. Entwicklungsstadium dieser Bestrebungen in groben Umrissen darzustellen.

Charakteristisch für alle kolloiden Lösungen ist, daß die disperse Phase gegen das Dispersionsmittel eine elektrische Potentialdifferenz hat. Rein physikalisch betrachtet kann man sich ihr Substrat als elektrische Doppelschicht im Sinne von $\mathrm{Quincke}$ und $\mathrm{Helmholtz}$ denken. Die eine Phase dieser Doppelschicht adhäriert fest an dem dispersen Teilchen, wir wollen sie deshalb die fixierte Schicht nennen. Die andere Phase der Doppelschicht ist in dem schon $z$ weifellos flüssigen und gegen die Grenzfläche verschieblichen Teil des Dispersonsmittels gelegen, wir wollen sie deshalb die vers shiebliche Schicht nennen.

Fragen wir nach dem materiellen Substrat dieser beiden Schichten, so können wir uns heute darunter nur Schichten von lonen denken. Die eine Schicht besteht offenbar aus positiven, die andere aus negativen Ionen. Wenn nun die eine dieser Schichten den kolloiden Teilchen fest anhaften soll, und die andere in endlicher Entfernung von derselben und gegen sie verschieblich sein soll, dann verhält sich das ganze disperse Teilchen gerade so, wie die Molekel eines in Wasser gelösten Elektrolyten, mit der einzigen Besonderheit, daß die elektrolytische Dissoziation sie nicht in zwei, drei oder vier einzelne Ionen, sondern in noch mehr Einzelteile zerlegt. Nehmen wir an, daß das kolloide Teilchen eine 'negative Ladung gegen das Lösungsmittel hat, so ist gewissermaßen das kolloide Teilchen einschließlich seiner Doppelschicht eine einzige Molekel des Elektrolyten, und seine elektrolytische Dissoziation besteht darin, daß sich gewi-sermaßen ein einz'ges negatives Riesenion mit zahlreichen Ladungseinheiten und auf der anderen Seite eine große Zahl von einzelnen positiven Ionen gewöhnlicher Art und Größe bildet. Nehmen wir z. B. an, daß die verschiebliche Lage der Doppelschicht aus $\mathrm{H}$-Ionen besteht, so verhält sich das Kolloid gewissermaßen so, als ob es eine vielfachwertige Säure sei, das Anion derselben ist das kolloide Riesenion. Die Kationen sind die gewöhnlichen $\mathrm{H}$-Ionen. Der einzige Unterschied gegen die Lösung einer gewöhnlichen Säure ist, daß die H-lonen und die Säureanionen nicht ordnungslos durcheinander gewürfelt sind, sondern die Anordnungen der Doppelschichten haben. Aber schon hieraus folgt, daß man sich die bewegliche Sihicht, welche von den $\mathrm{H}$-Ionen gebildet wird, nicht als eine wirkliche flächenhafie Schicht im mathematischen Sinne vorsteilen darf, welche die feinen kolloiden Kügelchen in endlicher Entfernung umgeben, vielmehr muB man sich, nach $\mathrm{G}$ o u y, diese $\mathrm{H}$ - Ionenschicht als ein Gebilde von einer gewissen Dicke vorstellen. Es bildet sich nicht eigentlich eine scharfe Sihicht von $\mathrm{H}$-Ionen um die Teilchen, sondern die Sache verhält sich nur so, daß die Konzentration der $\mathrm{H}$-Ionen in unmittelbarer Nähe der kolloiden Teilchen größer sein muß, als sonst durchschnittlich in der Lösung, und dies muß geschehen, um dem Gesetz der Elektroneutralität möglıchst Genüge zu leisten. Da die negativ geladenen Kolloidteilchen eine Anhäufung freier negativer Elektrizität bedeuten, muß in ihrer unmittelbaren Umgebung eine Anhäufung freier positiver Elektrızität stattfinden, welche dıe Negativität kompensiert. Daher ist also auch in bezug auf die Verteilung der freien .Elektrizi ätsmengen die kolloide Lösung inhomogener als die echte Lösung. Aber es handelt sich nur um einen quantitativen. Unterschied, denn auch, wenn wir uns eine echte Lösung eines Elektrolyten in genügend kleine Teilchen zerlegt denken, erscheint sie nicht mehr elektrisch homogen, sondern enthält abwechselnd Ttilchen mit freier positiver und Teilchen mit freier negativer Ladung. Kurz, es ist durchaus berechtigt, die disperse Phase einer kolloiden Lösung als einen Elektrolyten von etwas besonderer Natur $z u$ betrachten. 
Die Erscheinung der elektrischen Kataphorese erscheint alsdann einfach in dem Licht, daß das Kolloid wie jeder andere in der Lösung vorhandene Elektrolyt sich an der Stromleitung beteiligt. Hiermit aber haben wir einen elektrochemischen Gesichtspunkt für eine Systematik der Kolloide gewonnen, nämlich dadurch, daßs wir das Einteilungsprinzip der Elektrolyte auf die Kolloide übertragen.

Die Elektrolyte teilt man von jeher in einige Klassen ein und zwar zunächst in die Säuren und die Basen, je nachdem der Elektrolyt in wässeriger Lösung $\mathrm{H}$-Ionen oder $\mathrm{OH}$ Ionen abdissoziiert. Diejenigen Elektrolyte, welche je nach den Umständen sowohl $\mathrm{H}$-, wie $\mathrm{OH}$-Ionen abdissoziieren können, nennt man Ampholyte. Sämtliche anderen Elektrolyte, bei deren Dissoziation also die $\mathrm{H}$ - und $\mathrm{OH}$ Ionen nicht beteiligt sind, faßt man von jeher als $\mathrm{Sal}$ z e zusammen. Man kann sie entstanden denken als Reaktionsprodukte einer Säure und einer Base. Bei dieser Einteilung der Elektrolyte spielen also die $\mathrm{H}$ - und $\mathrm{OH}$-Ionen eine ganz besondere Rolle. Dieses Einteilungsprinzip ist uralt, ganz wesentlich älter, als die Theorie der Ionen, und rein aus Gesichtspunkten chemischer Reaktionsfähigkeit und Gegensätzlichkeit unterschied man von jeher Säuren, Basen und Salze.

Wollen wir also dieses Einteilungsprinzip auch für die Kolloidchemie zugrunde legen, so müßten wir sagen, die Kolloide werden eingeteilt in saure Kolloide, d. h. Kolloide, bei denen die bewegliche Schicht der Doppelschicht aus $\mathrm{H}$-Ionen besteht; in basische Kolloide, bei denen sie aus $\mathrm{OH}$-Ionen besteht; in amphotere Kolloide, bei denen je nach den Bedingungen das eine oder das indere der Fall sein kann, und schließlich als salzartige Kolloide, bei denen die bewegliche Schicht der Doppelschicht aus anderen Ionen als $\mathrm{H}$ - und $\mathrm{OH}$-Ionen besteht. $\mathrm{Da}$ wir aber diesen letzten Fall, wie in der gewöhnlichen Elektrochemie, uns dadurch entstanden denken können, daß die $\mathrm{H}$-Ionen durch Metallionen, oder die $\mathrm{OH}$-Ionen durch Säureanionen ersetzt sind, so kommt durch die Aufstellung der salzartigen Kolloide nichts prinzipiell neues in das System hinein.

Um diesen Gedankengang durchzuführen, müssen wir uns zunächst die Definition der Säuren und Basen noch einmal vergegenwärtigen. Wir können heute als Säure e ine in Wassermolekulardispers lösliche Molekelkraft definieren, welche in ein Anion und ein $\mathrm{H}$-lon $z u$ dissozileren vermag. Uebertragen wir diese Definition auf nicht dispers lösliche Stoffe, so fehlt hier das erste Kriterium der Säure, die molekulardisperse Löslichkeit. Da wir an feststehenden Begriffen lieber nichts ändern wollen, so wollen wir für den etwas abgeänderten Begriff auch lieber ein anderes Wort benutzen und wir definieren: ein Azidoid ist ein nicht molekulardispers löslicherStoff, welcher sich gegenüber reinem Wasser negativ lä dt, d. h. in Kombination mit den einzig heute möglichen Vorstellungen über die Natur dieser Ladung, welcher sich selbst wie ein negativ geladenes Riesenion verhält und von einer Schicht von $\mathrm{H}^{+}$-Ionen umgeben ist. Ganz entsprechend definieren wir das Basoid, indem. wir $\mathrm{OH}$-Ionen an Stelle von $\mathrm{H}$-Ionen setzen. Ein Ampholytoid ist ein nicht molekular löslicher Stoff, welcher je nach der H-Ionenkonzentration der umgebenden Lösung bald negativ geladen, und mit einer Schicht von $\mathrm{H}$-Ionen umgeben ist, bald positiv geladen und von einer Schicht von $\mathrm{OH}$-Ionen umgeben. ist. Es muß eine ganz bestimmte Konzentration von $\mathrm{H}$-Ionen geben, bei welcher ein Ampholytoid elektroneutral ist. Diese $\mathrm{H}$-Ionenkonzentration nennen wir nach Analogie der Definitionen der gewöhnlichen Ampholyte den is oelektrischen Punkt des Ampholyten.

Es ist nun zweckmäßig, diese Definition ein klein wenig zu modifizieren; den Vorteil davon wird man bald erkennen.

Zu diesem Zweck betrachte man zunächst einmal, in welcher Weise die Säurenatur eines Stoffes wie $\mathrm{CO}_{2}$ sich äußert. Man schreibt gewöhnlich:

$$
\mathrm{CO}_{2}+\mathrm{H}_{2} \mathrm{O} \rightarrow \mathrm{H}_{2} \mathrm{CO}_{3} \rightarrow \mathrm{H}^{\circ}+\mathrm{HCO}_{3}{ }^{\circ} \text {. }
$$

Um also die Definition der Säure anwenden zu können, nimmt man als primäre Reaktion die Bildung eines Hydrates an, welches erst die eigentliche Säure sei.

Man kann aber ebensogut schreiben:

$$
\mathrm{CO}_{2}+\mathrm{OH}^{\prime} \rightarrow \mathrm{HCO}_{3}^{\prime} \text {. }
$$

Wir können daher definieren: Eine Säure ist eine an sich elektroneutrale Molekelart, welche entweder $\mathrm{H}$-Ionen abdissoziiert, oder $\mathrm{OH}$-Ionen bindet, derart, daß die negative Ladung des $\mathrm{OH}$-lons erhalten bleibt. Diese beiden Vorgänge sind nicht unterscheidbar. Auch bei anderen Säuren, z. B. bei Essigsäure können wir die zweite Schreibweise durchführen:

$$
\mathrm{CH}_{3} \mathrm{COOH}+\mathrm{OH}^{\prime} \rightarrow \mathrm{CH}_{3} \mathrm{COO}^{\prime}+\mathrm{H}_{2} \mathrm{O} \text {. }
$$

Die undissoziierte Essigsäure addiert ein $\mathrm{OH}-\mathrm{Ion}$ und wird dabei eine Essigsäureanion 
unter Abspaltung von Wasser. Ganz analog wäre die Definition:

Ein Azidoid ist ein nicht molekulardisperser Stoff, welcher entweder H-Ionen abdissoziiert und dadurch negativ wird, oder welcher $\mathrm{OH}$-Ionen addiert, sei es mit oder ohne Abspaltung von Wasser, und dadurch ebenfalls negativ wird. Die Definition der Basen und Basoide ist ganz analog, wenn man überall $\mathrm{H}$ und $\mathrm{OH}$ miteinander vertauscht. Z. B. kann man die basische Natur des $\mathrm{NH}_{3}$ mit gleicher Berechtigung auf folgende zwei Weisen schreiben:

1. $\mathrm{NH}_{3}+\mathrm{H}_{2} \mathrm{O} \rightarrow \mathrm{NH}_{4} \mathrm{OH} \rightarrow \mathrm{NH}_{4}{ }^{\circ}+\mathrm{OH}^{\prime}$

2. $\mathrm{NH}_{3}+\mathrm{H}^{\circ} \rightarrow \mathrm{NH}_{4} \cdot$

Die Definition des Ampholyten und des Ampholytoiden folgt von selbst Jaraus. Der Dissoziationsgrad einer Säure hängt von der H-Konzentration der Lösung ab. Mit stejgender $\mathrm{H}$ geht die Dissoziation mehr und mehr zurück. Der Grenzfall ist, daß sie gleich 0 wird, aber niemals kann ein Säureion eine positive. Ladung annehmen. Uebertragen wir das auf die Azidoide, so würden wir sagen können:

Ein Azidoid wird durch steigende $[\mathrm{H}]$ des Lösungsmittels in seiner negativen Ladung mehr und mehr geschwächt. Der Grenzfall ist die Ladung 0 , es kann aber niemals durch noch so hohe Konzentration der $\mathrm{H}$-Ionen positiv umgeladen werden. Bei einem Basoid ist es ganz analog; es ist immer positiv geladen; und ein Ampholytoid wird durch eine bestimmte endliche H-Konzentration der Lösung entladen; oberhalb derselben ist es elektropositiv, unterhalb derselben negativ. Diese charakteristische $\mathrm{H}$-Konzentration ist der i so e le ktris che Punkt des Ampholytoids. Ich mache darauf aufmerksam, daß diese Definitionen sich nur auf die Beziehungen zum H-Ion erstrecken. Wir werden später sehen, daß Ampholytoide und sogar einige Azidoide auch durch mehrwertige Ionen $\left(\mathrm{Al}^{\cdots} \cdots\right)$ umgeladen werden können. Sie haben daher auch mit bezug auf $\mathrm{Al}$-Ionen einen isoelektrischen Punkt. Dieser aber ist hier nicht gemeint. Unsere jetzige, nur auf $\mathrm{H}$-Ionen bezügliche Definition des isoelektrischen Punktes $\left(\mathrm{J}_{\mathrm{b}}\right)$ ist unter der Voraussetzung gemacht, daß sich in der Lösung keinerlei solche dreiwertige, oder in anderer Weise aktive Ionen befinden, welche solche Stoffe umladen können.

Die Kataphorese und Endosmose gibt uns nun Gelegenheit, die Ladung der Kolloide zu studieren und $\mathrm{zu}$ entscheiden, welche Stoffe Azidoide usw. sind. Unter Benutzung der Versuche von Perrin, Glixelli, einiger Arbeiten aus meinem Laboratorium von Putter, Gyemant und $U m e t s u$, sowie von $B e t h e$ und To rop off, kann man folgendes sagen:

Azidoide sind: Kieselsäure, Mastix, Agar, Kollodium, Zuckerkohle, Retorienkohle, Edelmetalle, Zellulose, Bakteriensubstanz.

$B$ a s o ide sind bisher nicht bekannt; Stoffe basoider Natur haben, soweit sie bis jetzt untersucht worden sind, sämtlich einen isoelektrischen Punkt bei einer bestimmten $\mathrm{H}$-Konzentration und gehören daher zu den Ampholytoiden.

Ampholytoide sind: Aluminiumoxyd, Eisenoxyd, Eiweißstoffe verschiedener Natur, Gelatine, das Protoplasma der meisten Zellen, auch der Protozoen (im Gegensatz zu den Bakterien), Blutkohle.

Betrachten wir nun diese Stoffe, so weit sie chemisch einheitlicher Natur sind, so erkennen wir erstens darin solche Stoffe, denen wir aus ihrer chemischen Konstitution ohne weiteres eine reine Säurenatur zuschreiben würden (z. B. Kieselsäure, sowie Mastix, d. h. Harzsäuren). Bei diesen stimmt die auf Grund der Kataphorese definierte Natur als Azidoid mit der auf Grund der chemischen Zusammensetzung erwarteten Natur als Säure vollkommen überein. Andere Stoffe wiederum sind dabei, bei denen die rein chemisch erkennbare Ampholytnatur mit ihrem Verhalten als Ampholytoid bei der Kataphorese vollkommen übereinstimmt (Tonerde, Eisenoxyd, Eiweiß, Gelatine). Es sind aber mehrere andere Stoffe dabei, die wir rein chemisch nicht genau definieren können und nach ihrer Reaktionsfähigkeit für elektroindifferent halten würden. Sie sind fast alle Azidoide (Kollodium, Agar, Zuckerkohle, Retortenkohle), ein einziger von diesen verhält sich wie ein Ampholytoid, die Blutkohle, welche einen genau bestimmbaren isoelektrischen Punkt bei $\mathrm{p}_{\mathrm{H}}=3$ hat.

Wenn man sich eine Vorstellung davon machen will, wie ein chemisch indifferenter Stoff sich wie ein Azidoid verhalten kann, so können wir eine Hypothese zu Hilfe nehmen, welche nicht sehr gewagt ist und sich bisher gut bewährt hat. An Grenzflächen chemisch indifferenter Natur müssen die Adsorptionserscheinungen im eigentlichen Sinne am reinsten zum Ausdruck kommen. Bringen wir einen solchen Stoff in eine wässerige Lösung, so werden die verschiedenen Ionenarten zu Adsorptionserscheinungen Anlaß geben. Wir brauchen nun bloß anzunehmen, daß im allgemeinen die $\mathrm{OH}$-Ionen stärker adsorbiert werden als die $\mathrm{H}$-Ionen, um die azidoide Natur dieser Stoffe 
zu erklären. Uebrigens deckt sich die Annahme mit den Befunden der Balloelektrizität. Hier erweist sich der Nutzen der Definition, daß wir als Azidoid einen Stoff definiert hatten, welcher entweder $\mathrm{H}$-Ionen abdissoziiert, oder $\mathrm{OH}$-lonen bindet.

Für diese letzteren Azidoide "zweiter Art", wenn ich sie so nennen darf, kommt also nur die Adsorption von $\mathrm{OH}$-Ionen in Betracht, nicht aber die Abdissoziation von $\mathrm{H} \cdot$-Ionen. Mit dieser Annahme stimmt nun eine aufällige Erscheinung überein, welche bisher wenig beachtet worden ist.

Unter den Azidoiden gibt es nämlich einige, welche, wenn auch nicht durch $\mathrm{H}$-Ionen, so doch durch dreiwertige lonen von $\mathrm{Al}^{\cdots}$ positiv umgeladen werden können. Von den erwähnten Stoffen zeigen diese Eigenschaft Kieselsäure, Mastix u. a. Anderseits gibt es azidoide Stoffe, welche nicht einmal durch Al-Ionen positiv umgeladen werden, das sind Agar, Kollodium (wofern es nicht eiweißhaltige Körper adsorbiert enthält). Die ersteren sind offenbar Körper, welche $\mathrm{H}$-Ionen abdissoziieren können. Durch einen Ueberschuß von $\mathrm{H}$-Ionen wird diese Dissoziation zurückgedrängt, d. h. die $\mathrm{H}$-Ionen werden wieder gebunden, und es entsteht ein elektroneutraler Komplex. Befinden sich nun Al-Ionen in der Lösung, so können auch diese an Stelle der H-Ionen gebunden werden. Da die Al-Ionen aber mehrwertig sind, so kann es bei geeigneten Mengenverhältnissen eintreten, daß nur die ein e Ladung des Al-Ions neutralisiert wird, und die anderen frei bleiben. Dadurch wird der vorher elektronegative Körper positiv umgeladen. In die Sprache der Chemie übersetzt, würde das lauten: es bildet sich ein basisches Al-Salz. Dagegen bei den Azidoiden zweiter Art können $\mathrm{H}$-lonen gar nicht gebunden werden und daher ebensowenig Al-Ionen. Eine Umladung ist daher auch nicht durch Al-Ionen möglich.

Ein Mangel dieser Definitionen besteht vorläufig noch darin, daß sie nur qualitativer Natur sind. Bei den einfachen Säuren und Basen können wir nicht nur die Natur der Säure und Base qualitativ definieren, sondern auch ih re Stärke messen, indem wir in bekannter. Weise unter Anwendung des Massenwirkungsgesetzes die Dissoziationskonstante bestimmen. Die Anwendung des Massenwirkungsgesetzes ist nun für grobdisperse Stoffe im allgemeinen nicht möglich, und daher haben wir auch $z$. $Z$ t. noch keine'Methode, um die Stärke eines Azidoids zu definieren. Das ist Sache der Zukunft.
Diese Definition wird so beschaffen sein müssen, daß sie fúr den Grenzfall der molekularen Dispersion identisch wird mit der bekannten Definition der Stärke einer echten Säure. Nur gefühlsmäßig können wir vorläufig sagen: Ein Azidoid ist stark, wenn es auch in sehr saurer Lösung noch merklich negativ ist; es ist schwach, wenn es schon in mäßig saurer Lösung praktisch entladen wird.

Säuren und Basen können Salze bilden. Was ist die Analogie dazu bei den Azidoiden?

Wir müssen zwei Fälle unterscheiden, entweder ist die Base, mit der das Azidoid das Salż bilden kann, eine molekulardisperse Base, oder sie ist selbst ein Kolloid. Gestatten Sie mir, daß ich heute der Einfachheit halber nur den ersten Fall betrachte, dann aber ist die Definition der Salzbildung eines Azidoids sehr leicht. Die Salzbildung tritt dann ein, wenn die H-Ionen der einen Lage der Doppelschicht ersetzt werden durch Metallkationen. Bleiben diese Kationen in der verschieblichen Lage der Doppelschicht, so haben wir die Analogie zu einem total dissoziierten Salz. Werden sie aber fester gebunden, so ist diese Art der Salzbindung identisch einerseits mit der Entstehung undissoziierter Salzmolekel, anderseits mit der Ionenaustauschadsorption.

Beim Gespräch mit Fachgenossen über diese Gedankengänge ist ein Einwand aufgetaucht, der auf den ersten Augenblick bedenklich erscheint und deshalb hier vorgetragen werden soll.

Bekanntlich haben alle Stoffe von hoher Dielektrizitätskonstante gegen $W$ asser eine negative Ladung (Coehn'sche Regel), und zwar nicht nur solche Stoffe, denen wir ihre saure Natur aus ihrer chemischen Konstitution heraus ansehen, wie Phenol, sondern auch entschieden basische Stoffe wie Anilin. Das letztere haben insbesondere in letzter Zeit Freundlich und Gyemant bestätigt. Nun könnte man meinen, wenn Anilin in wässeriger Lösung eine Base ist, so müßte es in Form von Oeltröpfchen sich wie ein Basoid verhalten, also positiv geladen sein. Dieser Schluß trifft aber nicht $z u$, denn die positiv geladenen Anilinmoleküle sind molekulardispers in Wasser löslich. Es ist kein Grund vorhanden, warum die Oberfläche eines Anilintröpfchens mit Anilinionen bedeckt sein soll. Die Anilinionen verteilen sich homogen in Wasser. Bei einem Basoid dagegen müBte der Sachverhalt derart sein, daß die Ionen dieses Stoffes $\mathrm{nicht}$ molekulardispers in Wasser löslich sind, sondern zu einem Kolloidmizell verbunden bleiben. So ist es bei Mastix der Fall. Mastix 
ist ein Azidoid, weil folgende zwei Eigenschaften zusammentreffen: 1. ist es seiner chemischen Natur nach eine Säure, 2. sind die Anionen dieser Säure nicht in Wasser löslich, sondern bleiben an der Oberfläche des Mastixmizells adhärent, und nur die $\mathrm{H}$-lonen tendieren in die Lösung hinein. Die negative Ladung der Anilintröpfchen gegen Wasser kañn man nur dadurch erklären, daß die Grenzfläche von Anilin und Wasser sich durch Adsorption mit $\mathrm{OH}$ lonen anreichert. Demnach wäre Anilin, als Oeltröpfchen betrachtet, ein Azidoid, und zwar ein solches zweiter Ordnung, dagegen Anilin, so weit es in Wasser gelöst ist, einfach eine Base. Für den Begriff des Azidoids und Basoids ist also erforderlich, daB die Ionen, welche diese Stoffe liefern, nicht molekulardispers in Wasser löslich sind. Wir operieren hier also mit dem Begriff "wasserunlöslicher lonen"; und ich werde hoffentlich nicht auf Widerspruch stoßen, wenn ich $z$. B. die Ionen der Kieselsäure oder der Harzsäuren für wasserunlöslich halte. Die Ionen der Fettsäuren dürften den Uebergang bilden. Bei den niederen Fettsäuren sind die Ionen noch molekulardispers in Wasser löslich, bei den höheren sind sie es um so weniger, je länger die Kohlenstoffkette wird, und die Ionen bilden kolloide Mizellen. Man könnte bei einer Fettsäure mit langer Kohlenstoffkette vielleicht sagen: Ihr eines Ende, das mit der $\mathrm{COOH}$ Gruppe, tendiert zum Wasser, ihr anderes Ende klebt stärker an einem zweiten Fettsäuremolekül als am Wasser. Solch ein lon wäre sozusagen an dem einen Ende wasserlöslich, an dem anderen Ende wasserunlöslich. Eine solche Anschauung wird man nach den bekannten Untersuchungen von $\mathrm{Lang} \mathrm{m}$ u ir $\mathrm{r}$ über die Orientierung oberflächlich gelegener Moleküle heute wohl gelten lassen.

Zum SchluB möchte ich noch einige Worte sagen über die Anwendbarkeit des Massenwirkungsgesetzes auf gewisse Reaktionen, die man zur Kolloidchemie rechnen könnte. In einer Reihe von Arbeiten habe ich versucht, die Kinetik einiger Fermente nach dem Massenwirkungsgesetz zu behandeln; und dies ist insbesondere für die Invertase von so gutem Erfolge gewesen, daB bei ihren Reaktionen nicht nur ungefähr, sondern mit denkbar größter
Exaktheit. Dissoziationskurven, die nach dem Massenwirkungsgesetz berechnet wurden, herausgekommen sind. Diese Untersuchungen sind von einem Teil der Forscher günstig aufgenommen worden. Ein anderer Teil der Forscher lehnt sie aber prinzipiell ab, weil sie es für unberechtigt halten, das Massenwirkungsgesetz auf ein Ferment anzuwenden. Bei ihnen steht das Dogma fest, daB die Fermente sämtlich nicht molekulardispers sind. Dies aber ist doch noch nicht gesichert. Es gibt zweifellos Lösungen von Stoffen, die zwar nicht durch Pergament diffundieren, die auch sicherlich ein sehr großes Molekulargewicht haben, und dennoch sich der molekularen Dispersion zum mindesten stark annähern. Nach den Untersuchungen von $S$ örensen darf man dies wohl für das kristallisierte Eieralbumin nicht be$z w e i f e l n$. Die sehr merkliche Diffundierbarkeit vieler eiweißartiger Lösungen, sowie vieler Fermentlösungen ') spricht dafür, daß wir kein Recht haben, die Lösungen solcher Stoffe unter demselben Gesichtspunkte zu betrachten, wie etwa eine kolloide Lösung von Gold oder von Mastix. Hinzu kommt, daß durch die neueren Untersuchungen von Euler, und ganz besonders von Willstätter, die Molekulargröße wie auch die Mizellargröße der Fermente, wenn ich so sagen darf, doch merklich heruntergedrückt wird. Infolge der allgemeinen Unmöglichkeit, die Fermente rein darzustellen, verknüpfen wir mit der Vorstellung eines Fermentes jene Extrakte hochkolloider Natur, die wir im allgemeinen vor uns haben, wenn wir mit Fermentlösungen arbeiten. Ich bin überzeugt, da $B$ sich unsere Vorstellungen über die Kolloidalität einiger (natürlich nicht aller) Fermente gewaltig ändern werden, wenn wir erst die reinen Fermente in den Händen haben werden.

Mit diesen Schlußbemerkungen wollte ich zum Ausdruck bringen, daß ich davor warnen möchte, die oben entwickelten, für wirkliche Kolloide gedachten Ërörterungen ohne weiteres auf jede beliebige Eiweiß- oder Fermentlösung zu übertragen. Für diese kommen wir vorläufig noch ein gutes Stück weiter, wenn wir uns bemühen, die für echte Lösungen gültigen Gesetze auf sie anzuwenden.

1) Trypsin diffundiert sogar allmählich etwas durch eine Pergamentmembran! 